\title{
Effect of atorvastatin in combination with different oral antidiabetic medications on lipid parameters and glucose level
}

\author{
Mohammed N. Abed \\ Department of Pharmacology \& Toxicology, College of Pharmacy, University of Mosul, Mosul- Iraq
}

ABSTRACT $\quad \frac{\text { Received }}{25.6 .2012} \quad \frac{\text { Accepted }}{15.1 .2013}$

Background: Dyslipidemia is common in patients with type 2 diabetes mellitus and is regarded as independent risk factor for cardiovascular disease.

The aim of this study was to evaluate the efficacy and safety of atorvastatin for the treatment of dyslipidemia in type 2 diabetics using either metformin or glibenclamide as oral hypoglycemic agents.

Materials and methods: Fourty five type 2 diabetic patients diagnosed with dyslipidemia met the study criteria and received $20 \mathrm{mg}$ /day atorvastatin, then followed after 2 months. The various biochemical parameters studied included glycated hemoglobin (HbAlc), fasting serum glucose levels (FSG), total cholesterol (TC), triglycerides (TG), low-density lipoprotein (LDL) cholesterol, high-density lipoprotein (HDL) cholesterol, very low-density lipoprotein (VLDL) cholesterol and atherogenic index (AI).

Results showed that atorvastatin in both studied groups caused significant reduction in all measured parameters with an elevation in HDL, but this effect was obvious and more significant in patients using metformin than those using glibenclamide.

Conclusion: This study concluded that the combination of atorvastatin and metformin was more effective on lipid profile and glucose level than atorvastatin plus glibenclamide in type 2 diabetic patients with dyslipidemia.

Keywords: Dyslipidemia, types diabetes mellitus, atorvnstatin.

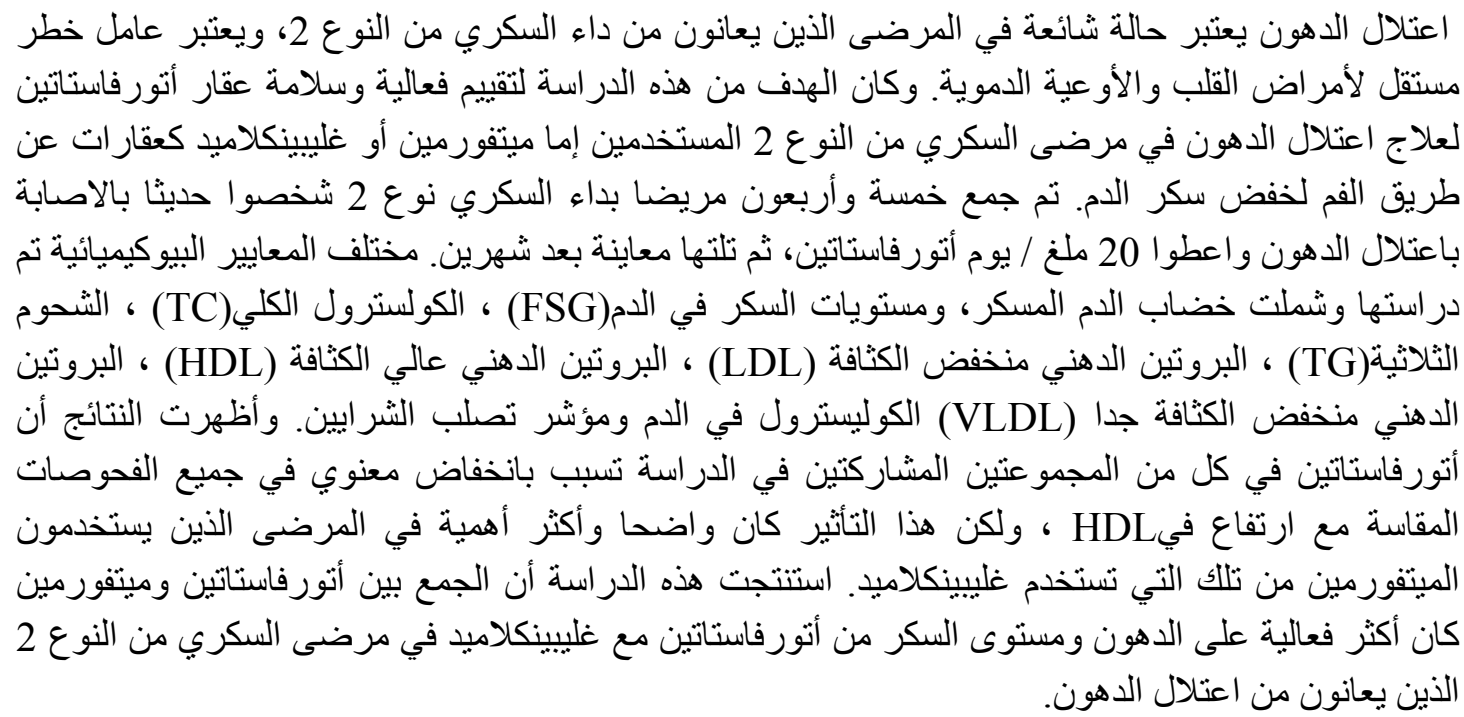


$\mathrm{T}$ ype 2 diabetes mellitus is associated with high morbidity and mortality from macro- and microvascular complications, one of the most dangerous complication is atherosclerosis which causes death in $75-80 \%$ of adults with type 2 diabetes ${ }^{1}$. Several other atherosclerotic risk factors are common in type 2 diabetes, like dyslipidemia, hypertension and obesity $^{2}$.

Dyslipidemia, an established risk factor for cardivascular disease (CVD), is common in patients with type 2 diabetes, affecting almost 50\% of this population ${ }^{3}$. In addition to hyperglycemia and hypertension, dyslipidemia is a modifiable CVD risk factor that remains largely uncontrolled in patients with type 2 diabetes $^{3}$.

In type 2 diabetes mellitus, dyslipidemia is usually presented in the form of increased triglycerides levels, decreased high density lipoproteins levels, and slightly higher low density lipoproteins (LDL) as compared to non diabetics ${ }^{4}$.

Aggressive lipid lowering therapy is essential in type 2 diabetic patients to reduce LDL, reduces cardiac mortality as well as total mortality ${ }^{5}$.

Statins are first line agents for the treatment of dyslipidemia in patients with type 2 diabetes mellitus, they effectively lower blood cholesterol levels and reduce the risk of cardiovascular events in many patients; also it has been shown that statins can reduce atherosclerosisrelated morbidity and mortality in patients with diabetes ${ }^{6}$. Statins also can improve other aspects of the lipid profile, such as increasing high-density lipoprotein cholesterol (HDL) and lowering LDL and triglyceride levels to some extent ${ }^{7,8}$.

Atorvastatin, which belongs to statins group, is a powerful HMG-CoA reductase inhibitor, proven to be safe ${ }^{9}$ and effective in reducing total cholesterol and triglycerides in dyslipidemic patients with hypercholesterolemia and primary hypertriglyceridemia ${ }^{10}$. This study was designed to see if treatment of dyslipidemia with atorvastatin in patients with type 2 diabetes mellitus affects glycemic control or serum lipid levels, and to show if there is any effect from oral hypoglycemic agents on the ability of atorvastatin in the treatment of dyslipidemia.

\section{Subjects, materials and methods}

Fourty five patients with type 2 diabetes mellitus on oral hypoglycemic therapy by either metformin or glibenclamide and had newly diagnosed dyslipidaemia were participated in this follow up study which was conducted from period between 1 st of December 2011 to the 1 st of June 2012.These patients underwent full history and complete physical examination. Exclusion criteria from the study included: Patients with Type 1 diabetes mellitus, Patients with type 2 diabetes mellitus using drugs other than metformin or glibenclamide, Patient with hepatic or ranal diseases and those with myocardial infarction, angina, and heart failure or hypertension because drugs that are used for treatment of these diorders may affect lipid profile.

According to the design of the study, patients were divided into two groups:

1- Group 1 (metformin group): included patients with type 2 diabetes mellitus treated by metformin this group consisted of 23 partients (11 male and 12 female), their mean age was (55.83 \pm 6.71 years).

2- Group 2 (glibenclamide group): included patients with type 2 diabetes mellitus treated by glibenclamide this group consisted of 22 partients (10 
male and 12 female), their mean age was $(58.09 \pm 8.70$ years).

Patient in both groups diagnosed as having dyslipidemia and they were kept on atorvastatin $20 \mathrm{mg} /$ day and followed after 2 months of therapy with this drug to assess their lipid parameters and glucose level.

Twenty five apparently healthy volunteers, who had no chronic disease and didn't receive any chronic medications, were also participated in this study as control group, they were 12 males and 13 females and their mean age was $56.72 \pm 7.03$ years.

Seven milliliters of blood for laboratory evaluation of clinical chemistry parameters were obtained from patients and control after an overnight fasting by antecubital vein puncture, $2.5 \mathrm{ml}$ of it were transferred into an anticoagulant EDTA tube with gentle shaking to obtain whole blood sample that was used for HbAlc measurement. The remaining blood was allowed to clot at room temperatures and after centrifugation the serum was collected in a plane tube and analyzed.

Serum total cholesterol, triglyceride, high density lipoprotein were measured according to procedures supplied by Biolabo company using optima UV-visible spectrophotometer. Low density lipoprotein (LDL) and very low density lipoprotein (VLDL) value was calculated by Friedewald formula (11). For LDL the formula is LDL-c $=\mathrm{TC}-$ HDL-c - TG/2.2, and for VLDL the formula is VLDL-c $=\mathrm{TG} / 2.2(\mathrm{mmol} / \mathrm{l})$. Atherogenic index was calculated according to the following equation: Athrogenic index $=$ total
cholesterol/HDL (12). Fasting serum glucose (FSG) was estimated by glucose - oxidase - peroxidase colorimetric method, by using commercial kit. HbAlc was measured in whole blood sample by ionexchange resin quantitative colorimetric determination.

Statistical methods: Data was expressed as mean \pm SD. Statistical analyses were done by using paired ttest to compare the measured parameters between before and after atorvastatin therapy, where as 2 samples t-test was used to compare between cases before and after telmisartan therapy with control. The statistical test results were considered significant at $\mathrm{p} \leq 0.05$.

\section{Results}

Treatment of dyslipidemia with atorvastatin in type 2 diabetic patients on metformin (group 1) resulted in significant reduction in all lipid parameters with significant increase in serum HDL and the percent was as followes: $\quad \mathrm{TC} \downarrow 25 \%, \quad \mathrm{TG} \downarrow 27 \%$, HDL $\uparrow 13 \%$, VLDL $\downarrow 27 \%$, LDL $\downarrow 34 \%$ and AI $\downarrow 35 \%$. Also the results showed that therapy with atorvastatin resulted in significant reduction in FSG and $\mathrm{HbAlc}$ at $\mathrm{p} \leq 0.05$ respectively (table 1 ). Treatment with atorvastatin in glibenclamide group (group 2) shoewd difference in reduction of lipid profile parameters which is less than that showed in group 1 and as followes: TC $\downarrow 14 \% ， \quad$ TG $\downarrow 23 \% ， \quad$ HDL $\uparrow 12 \%$, VLDL $\downarrow 23 \%$, LDL $\downarrow 19 \%$ and AI $\downarrow 24 \%$. FSG and HbAlc were also reduced to a significant level in this group at $\mathrm{p}<0.05$ respectively (table 1 ). 
Table 1. Effect of atorvastatin (20 $\mathrm{mg}$ / day) on lipid profile and glucose level after 2 months of treatment in both studied groups.

\begin{tabular}{|c|c|c|c|c|c|c|}
\cline { 2 - 7 } \multicolumn{1}{c|}{} & \multicolumn{3}{c|}{ Group 1 (metformin group) } & \multicolumn{2}{c|}{ Group 2 (glibenclamide group) } \\
(n=23) & \multicolumn{2}{c|}{ (n) } \\
\hline parameter & before & after & P value & before & after & pvalue \\
\hline TC (mmo/l) & $6.04 \pm 1.17$ & $4.52 \pm 1.22$ & $<0.001^{* *}$ & $6.05 \pm 1.39$ & $5.18 \pm 0.81$ & $<0.05^{*}$ \\
\hline TG (mmol/l) & $2.25 \pm 1.09$ & $1.65 \pm 0.66$ & $0.001^{* *}$ & $2.68 \pm 1.19$ & $2.04 \pm 1.18$ & $<0.05^{*}$ \\
\hline HDL (mmol/l) & $0.98 \pm 0.25$ & $1.13 \pm 0.22$ & $<0.05^{*}$ & $0.96 \pm 0.19$ & $1.1 \pm 0.29$ & $>$ \\
\hline VLDL(mmol/l) & $1.02 \pm 0.49$ & $0.73 \pm 0.26$ & $<0.05^{*}$ & $1.21 \pm 0.53$ & $0.92 \pm 0.53$ & $<0.05^{*}$ \\
\hline LDL (mmol/l) & $4.03 \pm 1.00$ & $2.65 \pm 1.11$ & $<0.001^{* *}$ & $3.87 \pm 1.5$ & $3.13 \pm 0.86$ & $<0.05^{*}$ \\
\hline AI & $6.16 \pm 1.62$ & $3.96 \pm 1.01$ & $<0.001^{* *}$ & $6.56 \pm 2.55$ & $4.99 \pm 1.31$ & $<0.05^{*}$ \\
\hline FSG (mmol/l) & $8.94 \pm 2.46$ & $7.84 \pm 2.03$ & $<0.05^{*}$ & $8.74 \pm 2.32$ & $7.35 \pm 1.43$ & $<0.05^{*}$ \\
\hline HbA1c \% & $7.54 \pm 1.33$ & $7.38 \pm 1.10$ & $0.05^{*}$ & $7.74 \pm 0.93$ & $7.53 \pm 0.88$ & $<0.05^{*}$ \\
\hline
\end{tabular}

Values are expressed as mean $\pm \mathrm{SD},{ }^{*}$ significant difference at $\mathrm{p} \leq 0.05$ and $* * *$ at $\mathrm{p} \leq 0.001$ using paired T test. TC total cholesterol, TG triglyceride, HDL high density lipoprotein, VLDL very low density lipoprotein, LDL low density lipoprotein, AI atherogenic index, FSG fasting serum glucose, HbA1c glycated hemoglobin.

Table 2. Comparison of lipid parameters and glucose level between control and each of patients before and after atorvastatin therapy in metformin group.

\begin{tabular}{|c|c|c|c|}
\hline prameter & $\begin{array}{c}\text { Control } \\
(\mathbf{n = 2 5})\end{array}$ & $\begin{array}{c}\text { Before atorvastatin } \\
(\mathbf{n = 2 3 )}\end{array}$ & $\begin{array}{c}\text { After atorvastatin } \\
(\mathbf{n = 2 3})\end{array}$ \\
\hline Total cholesterol (mmo/l) & $4.68 \pm 0.39$ & $6.04 \pm 1.17 * * *$ & $4.52 \pm 1.22$ \\
\hline Triglyceride (mmol/l) & $1.15 \pm 0.43$ & $2.25 \pm 1.09 * * *$ & $1.65 \pm 0.66^{*}$ \\
\hline HDL (mmol/l) & $1.41 \pm 0.35$ & $0.98 \pm 0.25^{* * *}$ & $1.13 \pm 0.22^{*}$ \\
\hline VLDL (mmol/l) & $0.52 \pm 0.19$ & $1.02 \pm 0.49^{* * *}$ & $0.73 \pm 0.26^{*}$ \\
\hline LDL (mmol/l) & $2.71 \pm 0.52$ & $4.03 \pm 1.00^{* * *}$ & $2.65 \pm 1.11$ \\
\hline Athrogenic index & $3.46 \pm 0.88$ & $6.16 \pm 1.62^{* * *}$ & $3.96 \pm 1.01$ \\
\hline FSG (mmol/l) & $4.85 \pm 0.7$ & $8.94 \pm 2.46$ & $7.84 \pm 2.03$ \\
\hline HbA1c \% & $5.46 \pm 0.26$ & $7.54 \pm 1.33^{* * *}$ & $7.38 \pm 1.10^{* * *}$ \\
\hline
\end{tabular}

Values are expressed as mean $\pm \mathrm{SD}$, *significant difference at $\mathrm{p} \leq 0.05$ and $* * *$ at $\mathrm{p} \leq 0.001$ using 2 samples $\mathrm{T}$ test. TC total cholesterol, TG triglyceride, HDL high density lipoprotein, VLDL very low density lipoprotein, LDL low density lipoprotein, AI atherogenic index, FSG fasting serum glucose, HbA1c glycated hemoglobin. 
Table 3. Comparison of lipid parameters and glucose level between control and each of patients before and after atorvastatin therapy in glibenclamide group.

\begin{tabular}{|l|l|l|l|}
\hline Parameter & $\begin{array}{l}\text { Control } \\
(\mathbf{n = 2 5})\end{array}$ & $\begin{array}{l}\text { Before atorvastatin } \\
(\mathbf{n = 2 2})\end{array}$ & $\begin{array}{l}\text { After atorvastatin } \\
(\mathbf{n = 2 2})\end{array}$ \\
\hline Total cholesterol (mmo/l) & $4.68 \pm 0.39$ & $6.05 \pm 1.39 * * *$ & $5.18 \pm 0.81^{*}$ \\
\hline Triglyceride (mmol/l) & $1.15 \pm 0.43$ & $2.68 \pm 1.19^{* * *}$ & $2.04 \pm 1.18^{*}$ \\
\hline HDL(mmol/I) & $1.41 \pm 0.35$ & $0.96 \pm 0.19^{* * *}$ & $1.1 \pm 0.29^{*}$ \\
\hline VLDL(mmol/l) & $0.52 \pm 0.19$ & $1.21 \pm 0.53^{* * *}$ & $0.92 \pm 0.53^{*}$ \\
\hline LDL (mmol/l) & $2.71 \pm 0.52$ & $3.87 \pm 1.5 * * *$ & $3.13 \pm 0.86^{*}$ \\
\hline Athrogenic index & $3.46 \pm 0.88$ & $6.56 \pm 2.55^{* * *}$ & $4.99 \pm 1.31^{* * *}$ \\
\hline FSG (mmol/l) & $4.85 \pm 0.7$ & $8.74 \pm 2.32^{* * *}$ & $7.35 \pm 1.43^{* * *}$ \\
\hline HbA1c \% & $5.46 \pm 0.26$ & $7.74 \pm 0.93^{* * *}$ & $7.53 \pm 0.88^{* * *}$ \\
\hline
\end{tabular}

Values are expressed as mean $\pm \mathrm{SD}$, *significant difference at $\mathrm{p} \leq 0.05$ and $* * *$ at $\mathrm{p} \leq 0.001$ using 2 samples $\mathrm{T}$ test. TC total cholesterol, TG triglyceride, HDL high density lipoprotein, VLDL very low density lipoprotein, LDL low density lipoprotein, AI atherogenic index, FSG fasting serum glucose, HbAlc glycated hemoglobin.

The statistical comparison of lipid parameter and glucose level between patients in metformin group before treatment with atorvastatin and control group showed a significant increase in all parameters at $(\mathrm{P}<0.001)$, but after treatment with atorvastatin TC, LDL and AI reached normal levels, other parameters reduced to lower values except for FSG and $\mathrm{HbA1c}$ which remain highly significat than control group (table2). On the other hand, the statistical comparison of lipid parameter and glucose level between patients in glibenclamide group before treatment with atorvastatin and control group showed a significant increase in all parameters at $(\mathrm{P}<0.001)$, and after treatment with atorvastatin the difference remain significant but at lower values except for FSG and $\mathrm{HbAlc}$ which remain highly significat than control group as shown in table 3.

\section{Discussion:}

In this study treatment with atorvastatin for 2 months in patient with type 2 diabetes mellitus was resulted in significant reduction in all lipid parameters in both studied groups, but although studied groups are matched for body weight and age, the comparison of results between them showed that atorvastatin was more effective when used in type 2 diabetic patients using metformin than those using glibenclamide.

There are no studies devoted exclusively to compare between the effects of lipid-lowering treatment on patients using diffeternt oral antidiabetic medications, however reports are available showing the merits of metformin therapy for a better control of lipid profile in type 2 diabetic patients ${ }^{13,14}$.

The mechanism by which atorvastatin affect lipid profile may be largely attributed to its ability in impairment cholesterol synthesis via inhibiting the enzyme HMG-CoA reductase which is the rate limiting step in cholesterol biosynthesis. This both decreases circulating lipoproteins and increases their uptake by up regulating hepatic LDL-C receptors. The overall lipid lowering effect include increase uptake and degrada- 
tion of LDL-C, inhibition of LDL-C oxidation, reduction in cholesterol accumulation and esterification and decreases lipoprotein secretion and cholesterol synthesis ${ }^{15}$.

Current recommendations for LDL goal is less than $2.58 \mathrm{mmol} / \mathrm{l}$ (an option of $<1.81 \mathrm{mmol} / 1$ in very highrisk patients), HDL goal greater than $1.03 \mathrm{mmol} / 1$ for men and greater than $1.29 \mathrm{mmol} / \mathrm{l}$ for women, an triglyceride goal is less than $1.69 \mathrm{mmol} / \mathrm{l}^{16}$. And also lowering LDL levels is the first priority in treating diabetic dyslipidemia because it is considered as an important cause of myocardial infarction or death from coronary heart disease is high ${ }^{17}$.

In this study, examination of the value of lowering LDL cholesterol level by atorvastatin in patients who have type 2 diabetes mellitus, showed that in metformin group $52 \%$ of patients reached the recommended LDL value compred to $31 \%$ in glibenclamide group, and this reduction is important to prevent CVD in these patients. Comparable results were obtained by Tan et al (18) who demonstrated the beneficial effect of $20 \mathrm{mg}$ of atorvastatin on endothelial function in type 2 diabetes with hypercholesterolemia, also analysis of results of many studies showed a significant decrease in the number of cardiovascular events in people with diabetes who received atorvastatin ${ }^{19-21}$.

$$
\text { Parameters characterizing }
$$
carbohydrate metabolism, like FSG and $\mathrm{HbAlc}$, in this study were increased significantly in both studied groups at baseline when compared with control, but after therapy with atorvastatin, FSG and $\mathrm{HbAlc}$ reduced significantly when compared with patients before atorvastatin administration, but these values are still higher than recommended because these patients have high mean values initially. These results are in consistent with results obtained from many studies that showed benificial effect of atorvastatin on glucose level in patients with type 2 diabetes mellitus ${ }^{22-24}$.

There is a strong consensus that reduction in HbAlc by any therapy (insulin or oral agents) diminishes microvascular complications. Metformin, however, is the only therapeutic agent that has been demonstrated to reduce macrovascular events in type 2 diabetes mellitus ${ }^{25}$, the result of this study may be of considerable value and pay attention for combination therapy between antidiabetic and hypolipidemic medications: therefore, the decreased effect of atorvastatin plus metformin therapy on FBS, HbAlc and lipid parameters in the present study may be to some degree of insulin resistance in patients with type 2 diabetes mellitus and this warrants the need for the introduction of insulin sensitizers (like metformin) in combination therapies for their success in future. Metformin may act both directly and indirectly to inhibit hepatic gluconeogenesis. In addition to its effect on the liver, metformin also appears to reduce glucose concentration by reducing peripheral insulin resistance and augmenting insulin mediated glucose uptake. Further metformin corrects dyslipidemia also $^{26}$, and this may explain the results obtained by the present study.

\section{Conclusion}

The antidiabetic drug metformin enhanced the hypolipidemic activity of atorvastatin more than glibenclamide, on the other hand atorvastatin enhanced the hypoglycemic activity of both drugs. These differential effects may be important in planning therapy when dyslipidemia accompanies type 2 diabetes because data obtained from this study suggested that combination of metformin and atorvastatin may be more benificial than combination of glibenclamide and atorvastatin in the 
treatment type 2 diabetic patients with dyslipidemia because it offers an advantage in terms of glycemic control and plasma lipid profile.

\section{References}

1. Laakso M. Epidemiology of Diabetic Dyslipidemia. Diabetes Rev 1995;3:408-422

2. Grundy SM, Hypertriglyceridemia, Atherogenic Dyslipidemia, and the Metabolic

syndrome. Am J Cardioi1998;81:18B25B.

3. Krishnaswami V. Treatment of dyslipidemia in patients with type 2 diabetes. Lipids in Health and Disease 2010, 9:144- 155

4. Imran A. Sheikh, Muzaffar A. Shaikh and Ikramudin Ujjan. Effect of atorvastatin and ezetimibe on dyslipidemia of type 2 diabetic patients.Medical channel 2010: Vol. 16, No. 2, 227229.

5. Poyorala K, Pedersen TR, Faergaman O, Olsson AG, thorgeirsson G. Cholesterol lowering with simvastatin improves prognosis of diabetic patients with coronary heart disease. A subgroup analysis of the Scandinavian Survival study(4s) Diabetes care 1997; 20:614- 20.

6. Vasilios G, Athanasios A, Valasia V, Dimokritos S, and Athanasios G. Atorvastatin and Micronized Fenofibrate Alone and in Combination in Type 2 Diabetes With Combined Hyperlipidemia. 2002; Diabetes Care 25:11981202.

7. Boden WE. Therapeutic implications of recent ATP III guidelines and the important role of combination therapy in total dyslipidemia management. Curr Opin Cardiol. 2003;18:278-285.

8. Expert Panel on Detection, Evaluation, and Treatment of High
Blood Cholesterol in Adults. Executive summary of the third report of the National Cholesterol Education Program (NCEP) Expert Panel on Detection, Evaluation, and Treatment of High Blood Cholesterol in Adults (Adult Treatment Panel III) JAMA. 2001;285:2486-2497.

9. Jong-Seon Park, Young-Jo Kim, JiYong Choi, Yoon-Nyun Kim, Teck-Jong Hong, Dong-Soo Kim, et, al. Comparative Study of Low Doses of Rosuvastatin and Atorvastatin on Lipid and Glycemic Control in Patients with Metabolic Syndrome and Hypercholesterolemia. Korean J Intern Med. 2010 March; 25(1): 27-35

10.Jones P, Laurora I and Hunninghake D. Comparative dose efficacy study of atorvastatin versus simvastatin, pravastatin, lovastatin, and fluvastatin in patients with hypercholesterolemia (the CURVES study). Am J Cardiol1998;81:582-87.

11. Friedewald WT, Levy R and Frederickson DS. Estimation of low density lipoprotein in plasma without use of the preoperative ultracentrifuge. Clin. Chem. 1972;18:499-502.

12. Goldberg IJ, (2001). Diabetic dyslipidemia: Causes and Consequences. Journal of clinical Endocrinology and Metabolism 8(3): 965-971.

13. Hermann LS, Karlson JE, Sijostrand A. Perspective comparative study in NIDDM using metformin and glibenclamide with special reference to lipid profiles. Eur J Clin 1991; 41: 263-5.

14. Purnell JQ, Hirsch IB. New oral therapies for type 2 diabetes. Am Family Physician 1997; 56: 1835.

15. Alegret M, Silvestre JS. Pleiotropic effects of statins and related 
pharmacological experimental approaches. Methods Find Exp Clin Pharmacol 2006; 28:627.

16. American Diabetic Association. Standards of medical care in diabetes 2006 diabetes care 2006;29(suppl 1) S4-S42.

17. Young SK Moon,Moti L. Pharmacological treatment of diabetic dyslipidemia Pharmacology Dec 2004 .24;12 1692-1713.

18. Tan KC, Chow WS, Tam SC, Ai $\mathrm{VH}$, Lam $\mathrm{CH}$. Atorvastatin lower C-reactive protein and improves endothelium- dependent vasodilation in type 2 diabetes mellitus. J Clin Endocrinol Metab 2002; 87: 563-8.

19. DALI Study: a double-blind, randomized, placebo-controlled trial in patients with type 2 diabetes and diabetic dyslipidemiaThe Effect of Aggressive Versus Standard Lipid Lowering by Atorvastatin on Diabetic Dyslipidemia. 2001Diabetes Care 24:1335-1341.

20. American Diabetes Association: Standards of medical care in diabetes- 2009. Diabetes Care 2009, 32(Suppl 1):S13-S61.

21. Carlos A. Aguilar-Salinas, Francisco J. Go'mez-Pe'rez a, Carlos Posadas-Romero b, Cuauhte'moc Va'zquez-Cha'vez c, Eduardo Meaney d, Alfonso Gulı'as-Herrero a, et, al. Efficacy and safety of atorvastatin in hyperlipidemic, type 2 diabetic patients. Atherosclerosis 2000: 152: 489-496.

22. Floriana E. I., Catalina P., Simona N., Rigas F. N., Mihat T., Florica P. Atorvastatin influence on glycemic control in patients with type 2 diabetes mellitus. Farmacia, 2010, Vol. 58, 6: 728-734.

23. Horvath E.M., Tackett L., Elmendorf J.S. - A novel membrane-based anti-diabetic action of atorvastatin. Biochem Biophys Res Commun, 2008, 372 (4), 639-643.

24. Koha K.K., Quonb M.J., Hana S.H., Leec Y., Kima S.J., Parka J.B., Shina E.K. Differential metabolic effects of pravastatin and simvastatin in hypercholesterolemic patients. Atherosclerosis, 2009, 204, 483490.

25. U.K. Prospective Diabetes Study Group. Effect of intensive bloodglucose control with metformin on complications in overweight patients with type 2 diabetes (UKPDS 34). Lancet, 1998b, 352:854-865.

26. Bell DSH, Mayo MS. Outcome of metformin facilitated reinitiation of oral antidiabetic therapy in insulin treated patients with NIDDM. Endocrine Practice 1997; 3: 73 . 